\title{
Using Breast Contour to Generate a Measure of Shape and Size Symmetry
}

\author{
Federico Cirett-Galán ${ }^{1 *}$, Raquel Torres-Peralta ${ }^{1}$, Valery Chernov ${ }^{2}$, Grigory Chernov ${ }^{3}$, José \\ Luis Ruiz-Duarte ${ }^{1}$ and Marcelino Barboza-Flores ${ }^{2}$ \\ ${ }^{1}$ Departamento de Ingeniería Industrial, Universidad de Sonora, Hermosillo, Sonora, Mexico \\ ${ }^{2}$ Departamento de Investigación en Física, Universidad de Sonora, Hermosillo, Sonora, \\ Mexico \\ ${ }^{3}$ Departamento de Física, Doctorado en Nanotecnología, Universidad de Sonora, Hermosillo, \\ Sonora, Mexico \\ fcirett@industrial.uson.mx
}

Breast cancer is the most common form of cancer in women worldwide; it represents $25 \%$ of all cancers in women. Infrared thermography of women breasts for early cancer detection is an approach that is not invasive and offers the opportunity to see for physiologic changes in the body years before other tools. An abnormal temperature distribution over a breast and usually its significant asymmetry could signal the existence of a malignancy. Another possible indicator of a tumor is a size and shape asymmetry between breasts.

It is a challenge to find the aforementioned asymmetry automatically in a thermal image, as a human body could be at a slight angle to the camera.

We approach this problem by differentiating both breasts' previously selected contours on a vector representation that allows to compare their size and shape using cosine similarity. Even though this is a Computer Vision problem, this framework requires low computing resources.

Our technique detects the small differences that appear in size and position of normal breasts, as there is no perfect symmetry in the human body, and it gives a measure of the difference between them that could be used in further applications using different criteria depending on the purpose.

Keywords: Breast cancer, infrared thermography, image processing, asymmetry, Computer Vision 